EPJ Web of Conferences 37, 01027 (2012)

DOI: $10.1051 /$ epjconf/20123701027

(C) Owned by the authors, published by EDP Sciences, 2012

\title{
The Crystal Ball programme at MAMI
}

\author{
D.P. Watts ${ }^{\mathrm{a}}$ for the CrystalBall@MAMI Collaboration \\ Institute for particle and nuclear physics, University of Edinburgh and SUPA, UK
}

\begin{abstract}
This contribution will outline the physics programme exploiting the intense energy tagged polarised real photon beam at MAMI-C. The coupling of an intense photon beam and close to complete detector acceptance offers unique opportunities for precision measurements to challenge our understanding of the structure of the nucleon and the nucleus as well as the underlying theory of Quantum Chromo Dynamics (QCD). The experimental facility and the physics programme will be outlined and selected recent preliminary analyses will be presented.
\end{abstract}

The proceedings are structured as follows. Section 1 outlines the MAMI-C microtron facility and the experimental apparatus in the real photon facility (A2) at MAMI-C. The scope of the real photon programme and some selected ongoing physics analyses will be outlined in section 2 .

\section{Experimental apparatus}

\subsection{The MAMI-C accelerator facility}

The MAMI-C[1] electron beam facility produces a $1.65 \mathrm{GeV}$ high quality $~ 100 \%$ duty factor electron beam from four cascaded racetrack microtrons. The layout of these mictotrons is shown in Fig. 1. The most recent of these using a novel harmonic double sided microtron design, was installed in 2005. This exploits four $90^{\circ}$ bending magnets with 2 linacs working at $2.45 \mathrm{GHz}$ and the first harmonic.

The MAMI-C electron beam can be passed to one of 4 experimental halls. The Crystal Ball [1] and TAPS [2] calorimeters are used in the real photon hall (A2 on Figure 1). In this hall the electron beam is converted to an intense $\left(\sim 10^{8} \gamma \mathrm{sec}^{-1}\right)$ beam of real photons through bremsstrahlung in a thin metal foil radiator. The scattered electrons following this process are momentum analysed in the Glasgow-Mainz Tagger, a magnetic dipole spectrometer with a highly segmented focal plane detector array [3]. This provides a determination of the energy of the associated bremsstrahlung photon with a resolution of 2-3 MeV and with time resolution $\sigma_{t}=0.117 \mathrm{~ns}$. Improved resolution and rate can be achieved for smaller sub ranges of photon energy by using a more highly segmented focal plane microscope sub-detector system [4], which makes use of the intrinsic $0.4 \mathrm{MeV}$ FWHM resolution of the tagger. The photon beam can be produced with a high degree of linear $(\sim 75 \%)$ or circular $(\sim 85 \%)$ polarisation.

The main tagger can tag photons up to $\sim 93 \%$ of the incident electron beam energy. To extend the energy range of the tagged photons a new endpoint tagger (EPT) has recently been commissioned. This is constructed from a new $\sim 1.2 \mathrm{~T}$ dipole magnet and associated focal plane detector array. The EPT is placed upstream of the current tagger (Fig. 2) and enables the analysis of low energy electrons associated with the higher energy photons close to the endpoint. This device extends the maximum achievable tagged photon energy at MAMI-C to $1.59 \mathrm{GeV}$. Importantly this allows measurements above the threshold for $f_{o}$ and $a_{0}$ mesons and increases the rate of tagged $\eta^{\prime}$ production.

\footnotetext{
a e-mail: dwatts1@ph.ed.ac.uk
} 

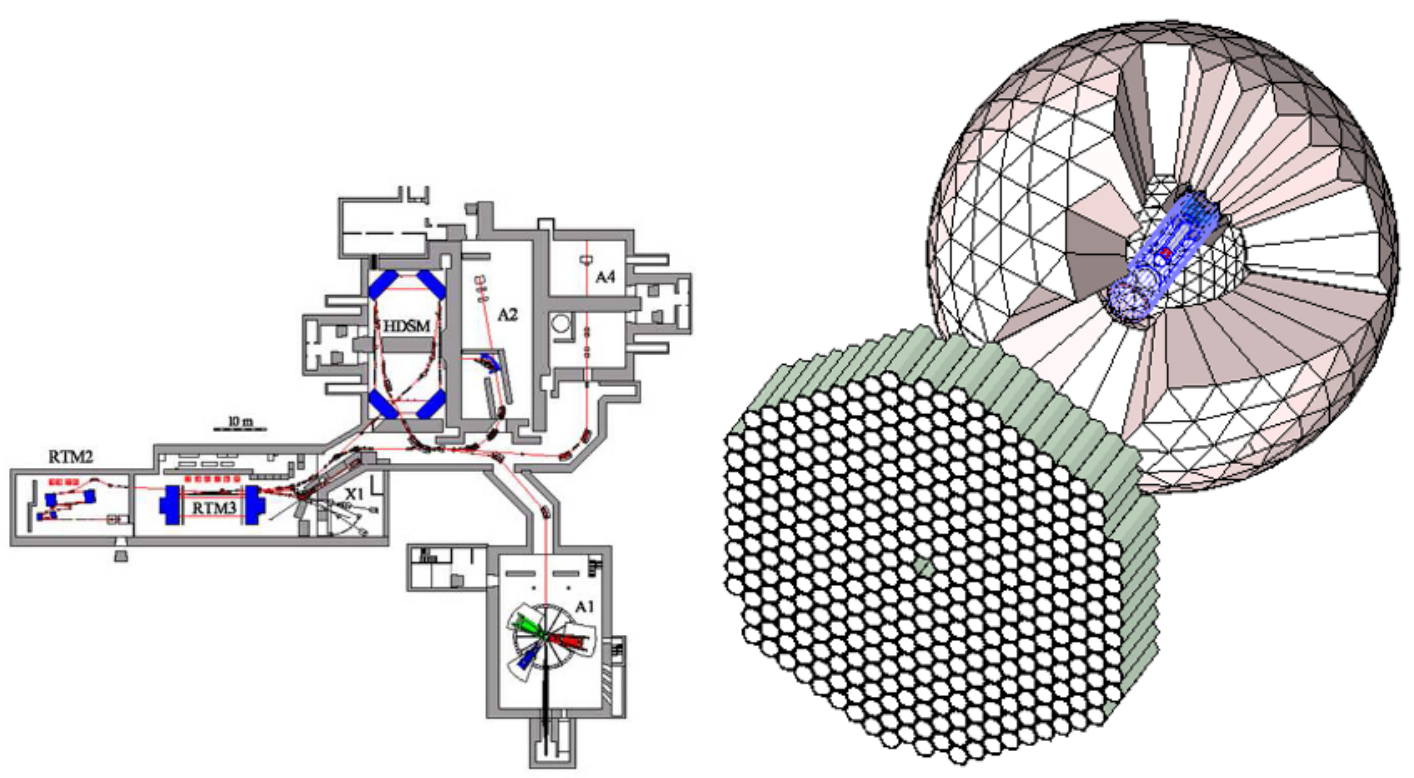

Fig. 1. Left: Layout of the accelerating microtron sections and the experimental halls at the MAMI accelerator facility. The real photon beam experiments are carried out in the A2 hall Right: Schematic figure showing the Crystal Ball and TAPS detectors. The PID detector is shown in blue and the target in red. The MWPC is located outseid the PID but has been omitted for clarity.

\subsection{Crystal Ball}

The Crystal Ball was conceived in the mid 1970s at SLAC and has been involved in experiments at SPEAR, DORIS and Brookhaven National Laboratory before arriving at MAMI. The Crystal Ball is a 672 element $\mathrm{NaI}$ detector covering $94 \%$ of $4 \pi$. Each element is shaped like a truncated pyramid $41 \mathrm{~cm}$ long (Figure 3). Photons incident on the ball produce an electromagnetic shower which generally deposits energy in a number of crystals (98\%) of the deposited energy of each photon is contained in a cluster of 13 crystals). From analysing the centre of gravity of the shower angular resolutions for the photon of $\sigma_{\theta}=2-3^{\circ}$ in theta and $\sigma_{\phi}=\sigma_{\theta} / \sin (\theta)$ in phi are achieved. The high light output of NaI also permits a good determination of the photon energy $\left(\sigma / \mathrm{E} \sim 1.7 \% / \mathrm{E}(\mathrm{GeV})^{0.4}\right)$.

A complete overhaul of the electronics for the Crystal Ball accompanied its move to Mainz. The scintillation light deposited in each crystal is read by its own 2 inch Photomultiplier tube (PMT). The output signal from each PMT is fed to a split-delay module. One output branch gives analogue sums of 4 or 16 crystals which can be used in trigger decisions. The second output branch of the split feeds individual multi hit TDCs and scalers via a dual threshold discriminator. The third output branch is fed to a sampling ADC (i-SADC108032) which samples the pulse shape at $40 \mathrm{MHz}$ (although an 80 $\mathrm{MHz}$ sampling capability which may be exploited in the future). The integral of the sampled signal is recorded for three time regions - before, during and after the scintillation pulse. This enables correction for remnant light present in the crystals before the event of interest to be corrected for, which improves the energy resolution.

The photon calorimetry capabilities of the Crystal Ball have been well established. The detector has had a more limited history in use for hadron calorimetry, but the low energy final state particles produced in many reactions at the Mainz beam energies makes development of this capability extremely desirable. The length of the counters corresponds to the stopping range of $233 \mathrm{MeV}$ for charged pions, $341 \mathrm{MeV}$ for charged Kaons and $425 \mathrm{MeV}$ for protons. For experiments at Mainz two sub-detector systems are placed inside the ball. The two multi wire proportional chambers (MWPCs) comprise 480 anode wires in the beam direction with 320 helically wound cathode strips. 

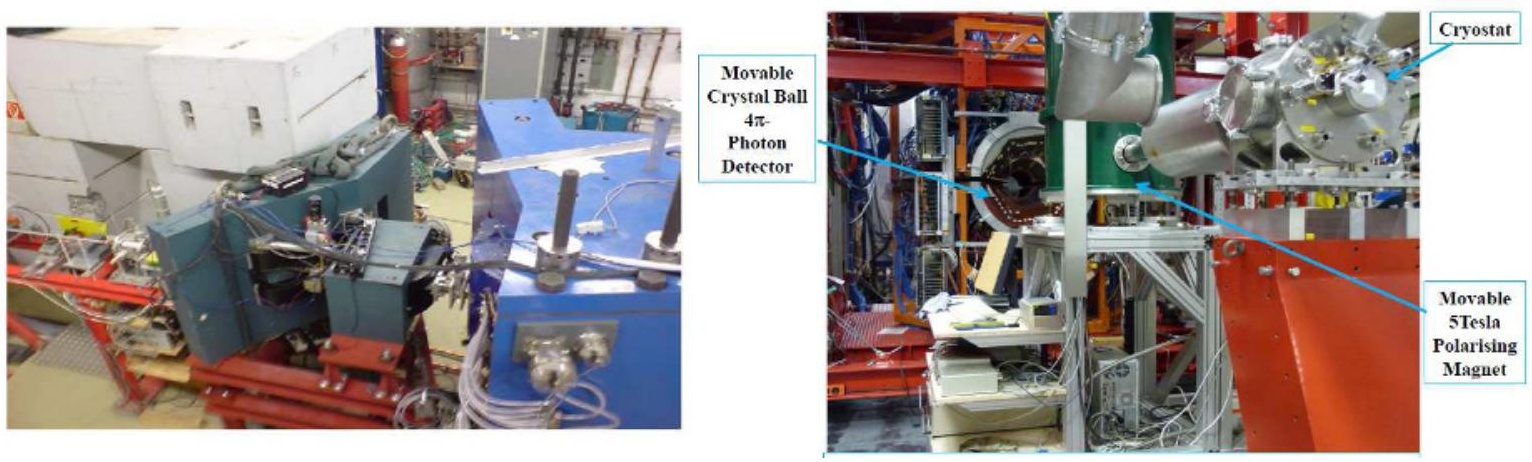

Fig. 2. Left: The new endpoint tagger constructed from the large dipole magnet on the left of the picture. The (blue) dipole from the existing photon tagger can be seen on the right. Right: The polarised target apparatus at MAMI. the target is in the polarising mode and is shown inserted in the 5T polarising magnet.

A $4 \mathrm{~mm}$ thick segmented plastic scintillator barrel provides a $\Delta \mathrm{E}$ signal with which to distinguish various charged particle types in the ball. Each scintillator element is $500 \mathrm{~mm}$ long with one side angled at 15 degrees so that the 24 scintillators can be formed into a barrel with minimal gaps between the scintillator edges. The scintillators are read out at one end by $9 \mathrm{~mm}$ diameter Hamamatsu Photonics R1635 photomultiplier tubes.

\subsection{TAPS BAF2 calorimeter}

The TAPS detector system covers the forward angle region for $\pm 20^{\circ}$ which is not covered by the Crystal Ball. This is an important part of the phase space for fixed target experiments with high beam momenta. TAPS consists of 510 hexagonally shaped BaF2 detectors each with diameter of $59 \mathrm{~mm}$ and $25 \mathrm{~cm}$ long, corresponding to 12 radiation lengths. BaF2 scintillator has two scintillation components, a fast component with a decay time of $0.6 \mathrm{~ns}$ and a slow component with a decay time of $620 \mathrm{~ns}$. Samples of the fast and slow component make possible pulse shape discrimination to give additional particle ID. Each TAPS crystal is covered by a $5 \mathrm{~mm}$ thick plastic scintillator tile which offers additional particle-ID capability by $\Delta E-E$ methods.

The 2 inner rings of TAPS have been replaced by $72 \mathrm{PbWO}_{4}$ crystals, each $20 \mathrm{~cm}$ in length corresponding to 22 radiation lengths. The higher granularity improves the rate capability in the region close to the beam where high electromagnetic backgrounds are experienced.

\subsection{Mainz/Dubna polarised target}

A major part of the ongoing and future programme at MAMI will exploit polarised nucleon targets. A new target system containing nucleons with a high degree of longitudinal or transverse polarisation has been developed. This consists of a large horizontal ${ }^{3} \mathrm{He} /{ }^{4} \mathrm{He}$ dilution refrigerator built in cooperation with Mainz and JINR Dubna (see Fig. 2). In the frozen spin technique the target material (butanol) is doped with small quantity of material producing free radicals. The electrons in the doped material are then highly polarised by cooling the target to milli Kelvin and placing it within a strong polarising field of 5 Tesla. Once the electrons are highly polarised their polarisation is transferred to the nucleons via Dynamic Nuclear Polarisation. In this process the transfer of spin is facilitated by exposing the target material to microwaves.

Once the spin is transferred the polarisation of the target is maintained by a smaller holding field provided by a superconducting coil placed around the target. During operation the target had a typical relaxation time of 1000 hours. The maximum polarisation for protons is $90 \%$. In the future the target will also operate with polarised deuterons from deuterated alcohol target material. 

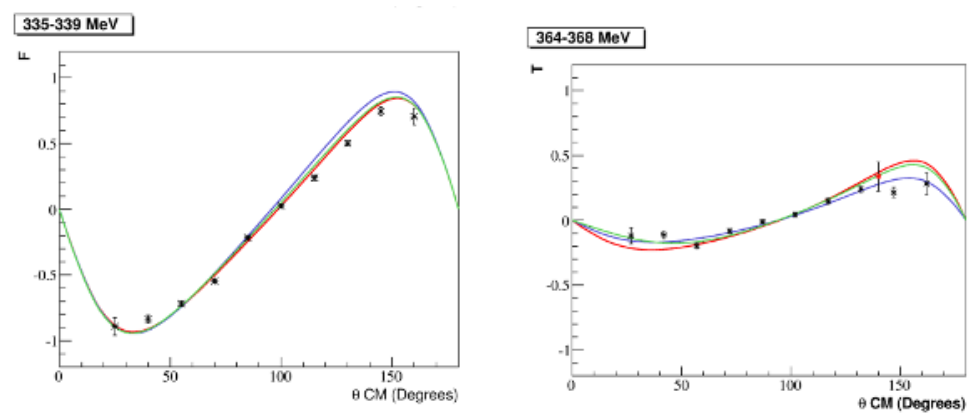

Fig. 3. Preliminary results for the $\mathrm{F}$ and $\mathrm{T}$ polarisation observables in $\pi^{0}$ photoproduction from the proton at the photon energies indicated in the figure. The lines represent di fferent partial wave analysis solutions.

\section{The Physics programme with the Crystal Ball at MAMI}

The intense tagged photon beam, polarised and unpolarised targets and close to complete detector acceptance enable a rich programme of precise physics measurements to be obtained. Some of the main aspects of the current programme at MAMI are indicated below

- Meson Physics Rare decays and tests of fundamental symmetries in $\eta$ and $\eta^{\prime}$ decays; Form factors (e.g. $\eta \rightarrow e^{+} e^{-} \gamma$ )

- Baryon Physics Polarisabilities of the nucleon $\left(\gamma N \rightarrow \gamma^{\prime} N\right)$; Establishing nucleon resonance properties (single and double meson photoproduction from the nucleon); Tests of low energy theorems such as Chiral Perturbation theory $(\gamma N \rightarrow N \pi$ near threshold)

- Nuclear /medium physicsMass shifts and line shape changes in the nuclear medium $(\gamma A \rightarrow$ $A($ meson $)$ ); Neutron skins of stable nuclei ( $\gamma A \rightarrow A \pi)$; Searches for mesic nuclei $(\gamma A \rightarrow A \eta)$

A few selected ongoing physics analyses are outlined below. For a comprehensive description of the future programme with the CrystalBall at MAMI the reader is referred to the recent successful Collaborative Research Centre (CRC) application for the MAMI facility which outlines the anticipated research priorities for the next 12 years.

\subsection{Polarisation observables in meson photoproduction}

The MAMI facility is in an excellent position to provide high quality polarised meson photoproduction data from the nucleon. Such data is very much needed to better constrain the partial wave analyses used to extract the properties and spectrum of nucleon resonances. Currently many nucleon resonances have poorly established properties such as mass, width and electromagnetic couplings. In pseudo scalar meson photoproduction from the nucleon 16 independent experimental observables are accessible. These comprise the cross section, single asymmetries from using polarised photon beam, target or recoiling nucleon and 3 quartets of double polarisation observables when 2 of beam, target or recoil polarisation are determined. There is a world effort underway to get as close to a "complete" measurement of observables as possible in meson photoproduction to better constrain partial wave analyses and enable precision determination of resonance properties and better challenge of non perturbative QCD predictions near to reaction thresholds.

An experimental campaign with a transversely a polarised proton target was carried out in 2010/11, comprising 5000 hours of beam on target. One part of the run focused on achieving high quality data for $\gamma\left(\mathbf{p}, \pi^{0}\right)$ in the region of the reaction threshold and the $\Delta$ resonance, with a second run using the full range of tagged photons available. The excellent quality of the $\gamma\left(\mathbf{p}, \pi^{0}\right)$ data for $\mathrm{T}$ (single polarisation observable for transversely polarised target) and $\mathrm{F}$ (double polarisation for circularly polarised photon beam and transversely polarised target) is evident from the preliminary data presented in figure 3 . It is clear the new data will be a very valuable contribution to the world data set. 
Incident sub-cluster at 3 ns

Decay sub-cluster at $20 \mathrm{~ns}$

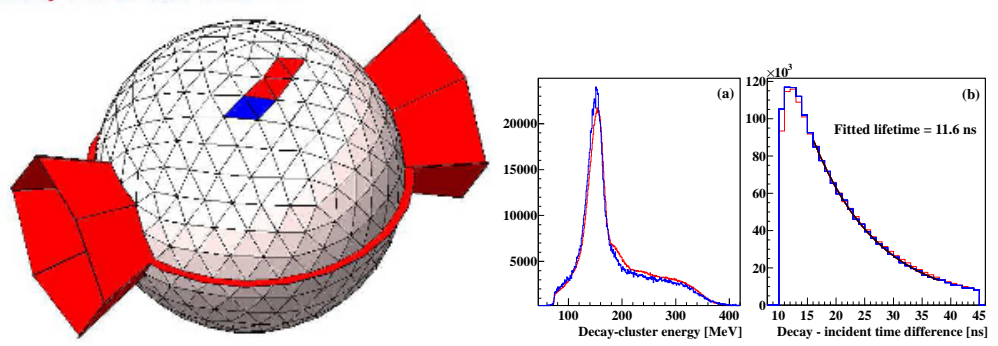

Fig. 4. Left: Schematic showing the typical cluster characteristics in the CB for a stopped Kaon decay. Middle and right: Decay-cluster characteristics for experimental and simulated data (blue and scaled red respectively). Middle is the Energy of the decay-cluster. Right is the time difference between incident and decay sub-cluster. An exponential fit yield a lifetime close to the expected $K^{+}$lifetime (inset).
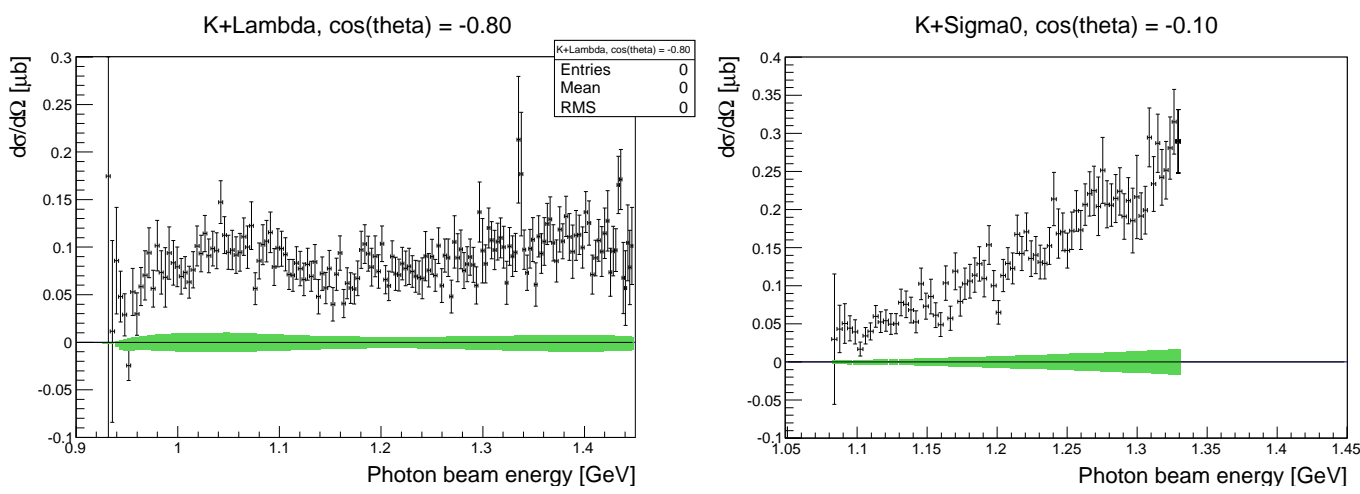

Fig. 5. Preliminary cross section data for $\mathrm{K} \Lambda$ (left) and $\mathrm{K} \Sigma$ (right) for the 2 centre of mass Kaon angle bins indicated on the figure. The green band shows the estimated systematic error

\subsection{Strangeness photoproduction}

As strangeness photoproduction reactions have cross sections typically orders of magnitude lower than non strange processes, even small contaminations from other reactions can spoil an event sample. With the Crystal Ball we have pioneered a novel method of $K^{+}$identification which cleanly identifies $K^{+}$ via its time delayed weak decay, $K^{+}$to $\mu^{+} v_{\mu}$ or $\pi^{+} \pi^{0}$ (pionic mode). A typical event is illustrated in fig. 4. The $K^{+}$lifetime of $12 \mathrm{~ns}$ was sufficiently long for the sub-clusters to be cleanly separated.

The validity of the new technique was tested extensively by comparing a full Geant 4 simulation of the apparatus with the experimental data. The results of some of these studies are presented in fig. 4. Panel (a) shows the energy of the decay-clusters, exhibiting a peak at $150 \mathrm{MeV}$ consistent with the $K^{+} \rightarrow \mu^{+} v_{\mu} \mu^{+}$decay at rest. The shoulder in the spectrum extends to the $350 \mathrm{MeV}$, which is the maximum energy deposition for the pionic decay. Panel (b) shows the accurate determination of the $K^{+}$lifetime from the spectra of time delays between the incident and decay-clusters.

Preliminary data for the $\mathrm{K}^{+} \Lambda^{0}$ and $\mathrm{K}^{+} \Sigma^{0}$ channels are shown in Fig. 5. For the photon energies accessible at MAMI-C the new data offers a factor of $\sim 5$ improvement in the invariant mass resolution and statistical accuracy compared to previous data. The $\mathrm{K}^{+} \Sigma^{0}$ data show very good agreement with the previous data from JLAB and SAPHIR. The new data for $\mathrm{K}^{+} \Lambda^{0}$ will be particularly valuable given the significant systematic discrepancies in this channel between the previous measurements. 

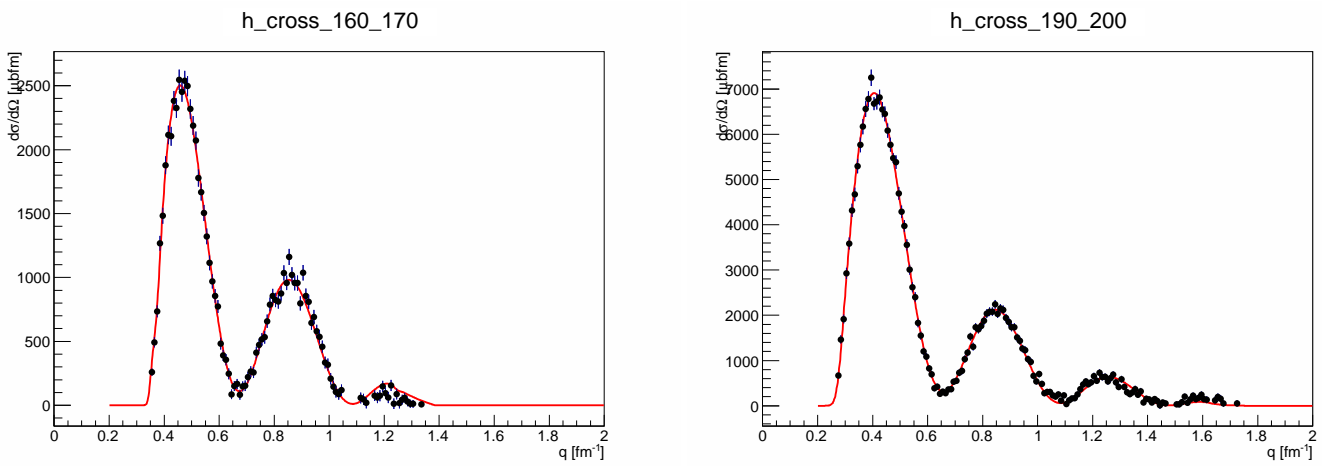

Fig. 6. Preliminary cross section data for coherent $\pi^{0}$ photoproduction from ${ }^{208} \mathrm{~Pb}$.Left: $E_{\gamma}=165 \pm 5 \mathrm{MeV}$. Right: $E_{\gamma}=195 \pm 5 \mathrm{MeV}$ The red line shows the theoretical model which has been interpolated to fit the data and find the best half radius and diffuseness for the neutron distribution.

\subsection{Coherent pion photoproduction}

The Crystal Ball and TAPS detector offer a highly efficient and largely uniform detection efficiency for $\pi^{0}$ mesons. This creates the possibility to measure coherent pion photoproduction processes from nuclei with sufficient precision to learn about the differences between the distributions of protons and nucleons in stable nuclei. The coherent $\pi^{\circ}$ reaction process proceeds with a close to equal probability on protons and neutrons and thus gives access to information on the matter form factor with an electromagnetic probe. The pion-nucleon interaction is strongest in the $P$-wave so by measuring the coherent reaction close to the threshold the produced pions have low momenta and the stronger effects from the pion-nucleus interaction are reduced.

Figure 6 shows the preliminary data from a ${ }^{208} \mathrm{~Pb}$ target for two of the incident photon energy bins. The red solid line in the figures show the predictions of the Coherent process [5]. In obtaining the predictions the charge distribution of lead has been fixed to that established experimentally but calculations obtained for a range of neutron half radii and diffuseness parameters. An interpolated fit to the experimental data has then been carried out to find the best radii and diffuseness parameters. In these fits consistent effects of a neutron skin with a preliminary value of $\sim 0.15 \mathrm{fm}$ are obtained. Further, the data are of sufficient quality that the diffuseness of the neutrons can also be extracted.

The new data will provide important constraints on nuclear structure calculations and help to constrain the equation of state (EOS) for neutron rich matter. The skin of a heavy nucleus shows a strong correlation with poorly established parameters of the neutron rich EOS.

\section{Summary}

The Crystal Ball and TAPS large acceptance calorimeter systems coupled with the intense tagged photon beam at MAMI provides an excellent facility to carry out precision studies and further our understanding of the structure of the nucleon, the nucleus and the underlying properties of non-perturbative QCD.

\section{References}

1. A. Starostin et. al., Phys. Rev. C64 055205 (2001)

2. R. Novotny et. al., IEEE Trans. Nucl. Sci 38374 (1991)

3. J.C. McGeorge et. al., Eur. Phys. Journ. A37 129 (2008)

4. A. Reiter et. al., Eur. Phys. Journ. A30 461 (2006)

5. D. Drechsel et. al., Nucl. Phys. A660 423 (1999) 